\title{
Conspecto das espécies brasileiras de Pseudachorutinae, com descrição de uma espécie nova da Colômbia (Insecta, Collembola)
}

\author{
Roger Arlé (*)
}

\begin{abstract}
Resumo
Vinte e duas espécies de Pseudachorutinae pertencentes à fauna brasileira são citadas. É dado um esquema da distribuição geográfica do grupo no Brasil de acordo com os nossos conhecimentos atuais. Duas espécies ocorrem praticamente em todas as regiōes naturais brasileiras, quatorze ocorrem na Amaônia, doze no Sudeste e seis no Nordeste. Uma espécie nova de Neotropiella Handschin, 1942, é descrita da região andina central da Colômbia (Páramo Purace 3000 m.alt.). E feito um relato de observações realizadas no litoral do Pará sobre a bionomia da espécie talassobionte Halachorutes schusteri Arlé, 1965, assim como sua reprodução e desenvolvimento em laboratório. Foi reencontrada a espécie Pseudanurida sawayana Schuster, 1965 num mangue do Recife. Outra espécie marinha Anurida maritima (Guérin), 1838 ocorre de Pernambuco até o litoral sul.
\end{abstract}

\section{INTRODUÇÃo}

Entre as subfamílias de Neanuridae (sensu Massoud 1967) é a subfamília Pseudachoruttnae que oferece maior diversidade nas regiões tropicais e subtropicais do hemisfério sul, e a complicada história de suas linhagens foi muito bem esclarecida nas suas grandes linhas por Massoud (1967). No que se refere ao Brasil são conhecidos 12 gêneros sendo alguns com tipo de distribuição africano-brasileiro como Kenyura, Arlesia, Arlesiella, Furculanurida e Aethiopella. É fora de dúvida que o grupo vai adquirindo importância biogeográfica cada vez maior com os progressos da pesquisa nas regiões tropicais.

As formas alargadas com paratergitos salientes, do tipo "Ceratrimeria" ou tipo 3 de Massoud (1967), com furca inoperante ou ausente (Brasilimeria), são espécies florestais, de hábitos cripticos ligadas aos biótopos saproxílicos. São de muita fraca mobilidade e geraimente de dispersão restrita, sendo também muito exigentes quanto à umidade e temperatura. Sendo a madeira decomposta um meio com excelentes qualidades reguladoras destes fatores, troncos em decomposição funcionam como verdacieiros micro-refúgios ecológicos estudacios por Delamare Deboutteville na África (Delamare, 1951). No decurso dos nossos estudos sempre encontramos pequenas famílias em reprodução sob cascas de troncos num determinado estado de decomposição e às vezes em galhos finos. Em trabalhos ante. riores publicamos algumas contribuições à ecolugia destas espécies (Arlé, 1939, 1943, 1959. 1966; Arlé \& Rufino, 1976).

Aqui convém tomar em consideração os paleoclimas que no quaternário, tanto na África como na América do Sul afetaram drasticamente as florestas equatoriais com alternâncias de períodos úmidos e secos, provocando a retração da mata equatorial reduzida a refúgios periféricos, seguida de novos avanços em períodos úmidos (Vanzolini, 1973). Estas grandes mudanças climáticas do quaternário com conseqüente deslocamentos das áreas florestadas, agindo sobre as antigas iinhagens gondwanianas de Pseudachorutinae, provocaram certamente especiações por segregação e iemanejamento coroiógico resuitando na atual distribuição do grupo.

Há indicações de que a recolonização da bacia amazônica se deu a partir de refúgios do planalto das Guianas (Tumucumaque) e refúgios andinos de um lado e planalto sul brasileiro do outro.

Atualmente os Pseudachorutinae têm no Brasil 2 centros principais de distribuição: a floresta amazônica e a floresta atlântica de

(") - Museu Nacional-Rio de Janeiro. Trabaiho realizado sob os auspicios do Conselho Nacional də Desenvolvimento Científico e Tecnológico, CNPq. 
encosta, onde algumas espécies como Brasili. meria wygodzinskyi, Ariesia fluminensis e Arlesia proxima são localizadas em altitude até 1500 ou $2000 \mathrm{~m}$.

Para as espécies talassobiontes de Pseudachorutinae (Halachorutes schusteri, Pseudanurida sawayana $\in$ Anurıda maritima) as condições que determinam a sua distribuição são evidentemente diversas, porém complexas. O litoral baixo do Pará (região bragantina), com grandes mangues, praias arenosas ou lodosas, é o domínio absoluto de Halachorutes schust€ri que ocorre em importantes populações. Nesta região porém não encontramos $P$ seucianurida sawayana nem Anurida maritima que ocorrem em localidades do Nordeste e Sudeste.

CONSPECTO DAS ESPÉCIES BRASILEIRAS

$$
\begin{aligned}
& \text { I - Tribu PSEUdAchorutinI Boerner } \\
& \text { (sensu Massoud). }
\end{aligned}
$$

Genus Pseudachorutes Tullberg, 1871 Pseudachorutes massoudi Arlé, 19ób Somente conhecido da mata de terra firme de Manaus.

Pseudachorutes herberti Arlé e Rufino, 1976.

Até o presente só conhecido de $\mathrm{Ma}$ naus, em húmus de mata de inundação (Rio Cuieiras) .

Genus Kenyura Salmon, 1954

Kenyura porculus (Arlé), 1959

Esta espécie só foi encontrada na Floresta da Tijuca, Rio de Janeiro.

Kenyura xinguensis Arlé, 1966 Parque indígena do Xingu (Alto Xingu). Em "litière" úmida de mata ciliar.

Kenyura delicata Arlé, 1966

Mata de terra firme de Manaus.

Genus Hylaeanura Arlé, 1966 Hylaeanura infima (Arlé), 1959

Descrita do Alto Xingu, parece ocorrer em toda a Amazônia pois a encontramos $\mathrm{em}$ numerosas localidades da Amazônia oriental, central e meridional. Foi assinalada do Peru amazônico (Winter 1963).
Genus Arlesia Handschin, 1942

Arlesia albipes (Folsom), 1927

É espécie de larga distribuição desde Costa Rica até o Sul do Brasil. É freqüente no Rio de Janeiro e na Amazônia.

Arlesia albipes f. decorata A.rlé e Rufino, 1979

Forma somente conhecida de Recife (Pernambuco) .

Arlesia fluminensis (Arlé), 1939

Espécie restrita à região de Petrópolis e Serra dos órgãos entre 800 e 1200 m de altitude.

Arlesia proxima (Arlé), 1939

Limitada à Serra do Mar na altura de Mangaratiba.

Genus Arlesiella Delamare Deboutteville, 1951 Arlesiella amazonica Arlé, 1966

Mata de terra firme de Manaus. Reserva Ducke, Colônia Sto. Antônio. Em "litière".

Genus Neotropiella Handschin, 1942

Nectropiella carli (Denis), 1924

Espécie muito disseminada em todo o norte da Amazônia, do Amapá a Manaus e até o Peru, Guianas e provavelmente Venezuela. É nossa opinião que N. araguaensis Rapoport, 1969, é sinônimo de $N$. carli. Não a encontramos na Amazônia meridional. No Pará seu limite sul ocorre na região de Belém onde é comum e onde se encontra esporadicamente $N$. meridionalis aqui no seu limite setentrional. Ao sul de Belém só encontramos $N$. meridionalis.

Neotropiella meridionalis (Arlé), 1939 É a Espécie típica do Sudeste, freqüente na Serra do Mar e no Rio de Janeiro (maciço da Tijuca). Como acabamos de mencionar ela alcança a Amazônia oriental na regiäo de Belém onde chega a coexistir com $N$. carli mas não aparece mais ao norte.

Neotropiella. quinqueoculata (Denis), 1931

É a espécie de maior distribuição na região nectropical e é muito variável. Ocorre em todo o Brasil. Foi estudada 

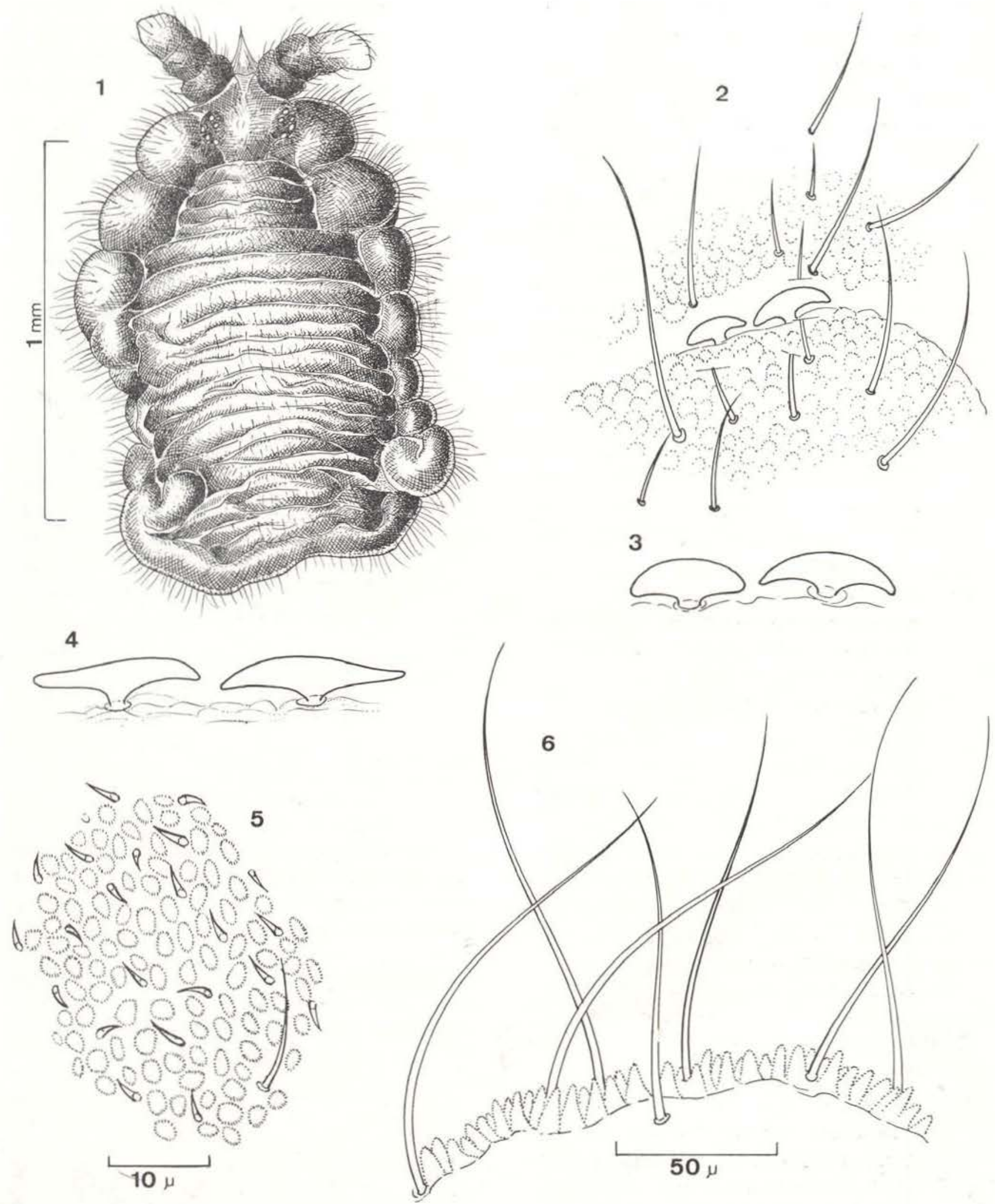

Figs. 1-6 - Neotropiella malkini sp.n.: 1) - Habitus; 2) - Orgão Ant. III; 3) - Papilas do órgão Ant. III; 4) - Idem Exemplar maior; 5] - Face inferior de Ant. IV; 6) - Cerdas dos paratergitos. 
por Lawrence (1971) sobre material de Mato Grosso Royal Society Expedition. Lawrence neste trabalho fez uma revisão das espécies de Neotropiella e demonstrou a grande variabilidade do órgão pós-antenal em $N$. quinqueoculata.

Neotropielia denisi (Arlé), 1939

Descrita originariamente de diversas localidades baixas do Rio de Janeirc esta espécie foi por nós encontrada no Alto Xingu em cerradão e áreas úmidas de mata, em VI - 1961. Os exemplares do Xingu porém possuem 7 olhos em vez de 6 de cada lado (fig. 15,16 e 17 ).

Genus Aethiopella Handschin, 1942

Aethiopella delamarei Arlé, 1959

Sómente encontrada no Sudeste, na Serra da Mantiqueira, em húmus profundo de capoeira em altitude de $900 \mathrm{~m}$.

Genus Brasilimeria Stach, 1949

Brasilimeria anura (Arlé), 1939

Freqüente na Serra do Mar nos arredores do Rio de Janeiro.

Brasilimeria wygodzinskyì (A.rlé), 1943 Próxima morfologicamente de $\mathrm{H}$. anura, esta bonita espécie que se distingue nos indivíduos suficientemente desenvolvidos por um padrão caracteristico de desenhos amarelos, parece substituir B. anura na Serra da Mantiqueira (Itatiaia, Barreira de Piquete, Itajubá, Campos do Jordão) em altitudes entre médias e altas $(500-1800 \mathrm{~m})$.

Genus Furculanurida Massoud, 1967

Furculanurida belemensis Arlé e Rufino, 1976

Belém - Utinga (várzea do Guamá).

Furculanurida goeldiana Arlé e Rufino, 1976

Estas duas espécies divergem pelo número de olhos ( 5 e 7 respectivamente) e pela forma da mandíbula. São conhecidas sómente da região de Belém sendo que $F$. goeldiana foi encontrada exclusivamente no Parque do Museu Goeldi.
Genus Halachorutes Arlé, 1966

Halachorutes schusteri Arlé, 1966

É espécie muito abundante em todo litoral do Pará e também encontrada em pequenas populações até o Rio de Janeiro.

Genus Pseudanurida Schott, 1901

Pseudanurida sawayana Schuster, 1965 Reencontramos esta espécie no canal da Lagoa do Olho d'água, no Recife em 1969. Schuster também a encontrou em Cananéia (litoral de São Paulo) .

$$
\begin{aligned}
& \text { II - Tribu Anurıdini (sensu Massoud } \\
& \text { 1967) }
\end{aligned}
$$

Genus Anurida Laboulbène, 1865

Anurida maritima (Guérin-Ménneville), 1838

Ė espécie talassobionte de ampla distribuiçăo peló mundo, é encontrada em quase toda a Europa. América do Norte atlântica, Africa do Sul e Brasil Nordeste e Sul onde foi estudada por Schuster (1965). Nunca a encontramos no litoral do Pará.

Para uma visẩo conjurita da distribuição da subfamilia no Brasil ver a tabela I.

\section{DESCRIÇÃO DE NOVA ESPÉCIE}

\section{Neotropiella malkini sp. nov.}

MATerial examinado: Páramo Purace Cordilheira Centra! (Depto. Cauca) $3000 \mathrm{~m}$ altitucie - Colômbia. Borys Malkin col. 2 exemplares $20 / 10 / 1968$.

DIAGNOSE: 2 exemplares de 1,50 e 2,10 $\mathrm{mm}$ respectivamente. Cor azul preto. Extremidades das patas e cone bucal esbranquiçados. Antenas com os segmentos III e IV amarelos.

Habitus típico de Pseudachorutini do tipo 3 (Massoud 1967). Pretergitos marcados, paratergitos muito salientes, aspecto geral fortemente enrugado (Fig. 1). Corpo revestido regularmente de cerdas bastante compridas, entre 100 e 150 micra [Fig, 6].

Antenas curtas e maciças. Uma papila apical trilobada e tubos sensoriais na face dorsal 
TABELA I

\begin{tabular}{|c|c|c|c|c|c|}
\hline Espácies & 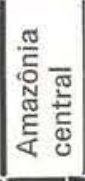 & 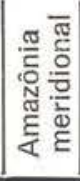 & 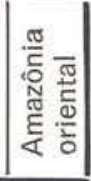 & 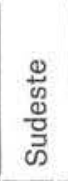 & 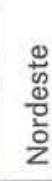 \\
\hline Kenyura xinguensis & & $\mathrm{x}$ & & & \\
\hline Kenyura delicata & $\mathrm{x}$ & & & & \\
\hline Kenyura porculus & & & & & $\mathrm{X}$ \\
\hline Pseudachorutes massoudi & $\mathrm{X}$ & & & & \\
\hline Pseudachorutes herberti & $\mathrm{x}$ & & & & \\
\hline Arlesia albipes & $\mathrm{X}$ & $\mathrm{X}$ & $\mathrm{X}$ & $\mathrm{X}$ & $\mathrm{X}$ \\
\hline Arlesia albipes f. decorata & & & & $\mathrm{X}$ & \\
\hline Arlesia fluminensis & & & & & $\mathrm{x}$ \\
\hline Arlesia proxima & & & & & $\mathrm{X}$ \\
\hline Arlesiella amazonica & $x$ & & & & \\
\hline Hylaeanura infima & $\mathrm{x}$ & $\mathrm{x}$ & $\mathrm{X}$ & & \\
\hline Neotropiella carli & $\mathrm{x}$ & $\mathrm{x}$ & $\mathrm{X}$ & & \\
\hline Neotropiella quinqueoculata & $x$ & $x$ & $\mathrm{x}$ & $\mathrm{x}$ & $\mathrm{x}$ \\
\hline Neotropiella meridionalis & & & $x$ & $?$ & $\mathrm{x}$ \\
\hline Neotropiella denisi & & $\mathrm{X}$ & & & $\mathrm{X}$ \\
\hline Furculanurida belemensis & & & $\mathrm{x}$ & & \\
\hline Furculanurida goeldiana & & & $x$ & & \\
\hline Aethiopella delamarei & & & & & $\mathrm{X}$ \\
\hline Brásilimeria anura & & 3 & & & $\mathrm{X}$ \\
\hline Brasilimeria wygodzinskyi & & & & & $\mathrm{X}$ \\
\hline Halachorutes schusteri & & & $\mathrm{X}$ & $\mathrm{X}$ & $\mathrm{X}$ \\
\hline Pseudanurida sawayana & & & & $\mathrm{X}$ & $\mathrm{X}$ \\
\hline Anurida maritima & & & & $x$ & $\mathrm{X}$ \\
\hline
\end{tabular}

de Ant. IV (Fig. 13). Face ventral de Ant. IV com cerclas muito curtas espalhadas entre as granulações, não formando revestimento denso. Órgâo antenal III formado por 2 tubérculos em forma de franquisque, numa leve dobra, mas descobertos (Fig. 2, 3 e 4); a fig. 4 representa o órgão antenal III do exemplar maior cujcs tubérculos têm forma um pouco mais achatada.

Grupo ocular de 5 olhos bem desenvolvidos e órgão pós-antena! moruliforme com tubérculos alongaods em forma de banana (Figs. $8 \mathrm{e}$ 9). O diâmetro do pós-antenal é ligeiramente inferior ao diâmetro de um olho. Cone bucal ponteagudo. Maxilas estiliformes. Mandibulas muito alongadas e praticamente estiliformes, com apenas um pequeno dente sub-apical (Fig. 7 e 10).
Unha com forte dente lateral e crista interna lisa, com muito pequeno dente basal.

Furca muito curta, mucro triangular, subigual à dens cuja face dorsal possui 5 cerdas bem desenvolvidas e 2 basais muito curtas na face interna (Fig. 11).

AFINIDADES: Neotropiella típica, esta espécie é perfeitamente caracterizada por uma série de particularidades como: forma do órgão antenal III com as suas papilas em forma de franquisque. Revestimento do ccrpo de cerdas longes, peças bucais com mandíbulas muito finas e simples, órgão pós-antenal com tubérculos em forma de banana e furca curta.

HOLÓtIPo: exemplar dissecado em lâmina procedente do Páramo Purace (Colômbia), Borys Malkin col. 20/10/68. Nas coleções do Museu Nacional - Rio de Janeiro.

OBSERVAÇÕES SOBRE AS ESPÉCIES TALASSOBIONTES

Nas costas baixas e muito recortadas do Pará, com imensos mangues, partes rochosas com biocos de arenito ferruginoso e pequenas falésias calcárias assim como praias arenosas ou iodosas, ocorre em abundância a espécie talassobionte Haiachorutes schusteri, porém não ocorre Anurida maritima, fato difícil de explicar pois Halachorutes schusteri coexiste com Anurida maritima, embora em pequenas colônias, no facies rochoso do litoral sudeste. Anurida maritima freqüenta unicamente as costa rochosas e relativamente abrigadas e é encontrada em grandes populações quando condições favoráveis são realizadas, como em algumas localidades do Nordeste e principalmente no litoral dos Estados de Espírito Santo, Rio de Janeiro e São Paulo. Devido à sua enorme distribuição que abrange quase todas cos. tas da Europa, foi um dos colêmbolos talassobiontes mais estudado (Laboulbène, 1864, Imms, 1906, etc.). No Brasil foi muito bem estudado por Schuster (1965) na litoral ae São Paulo e Recife.

Halachorutes schusteri por nós descrito de Salinópolis (Pará), foi encontrado posteriormente em grande quantidade nos mangues an- 


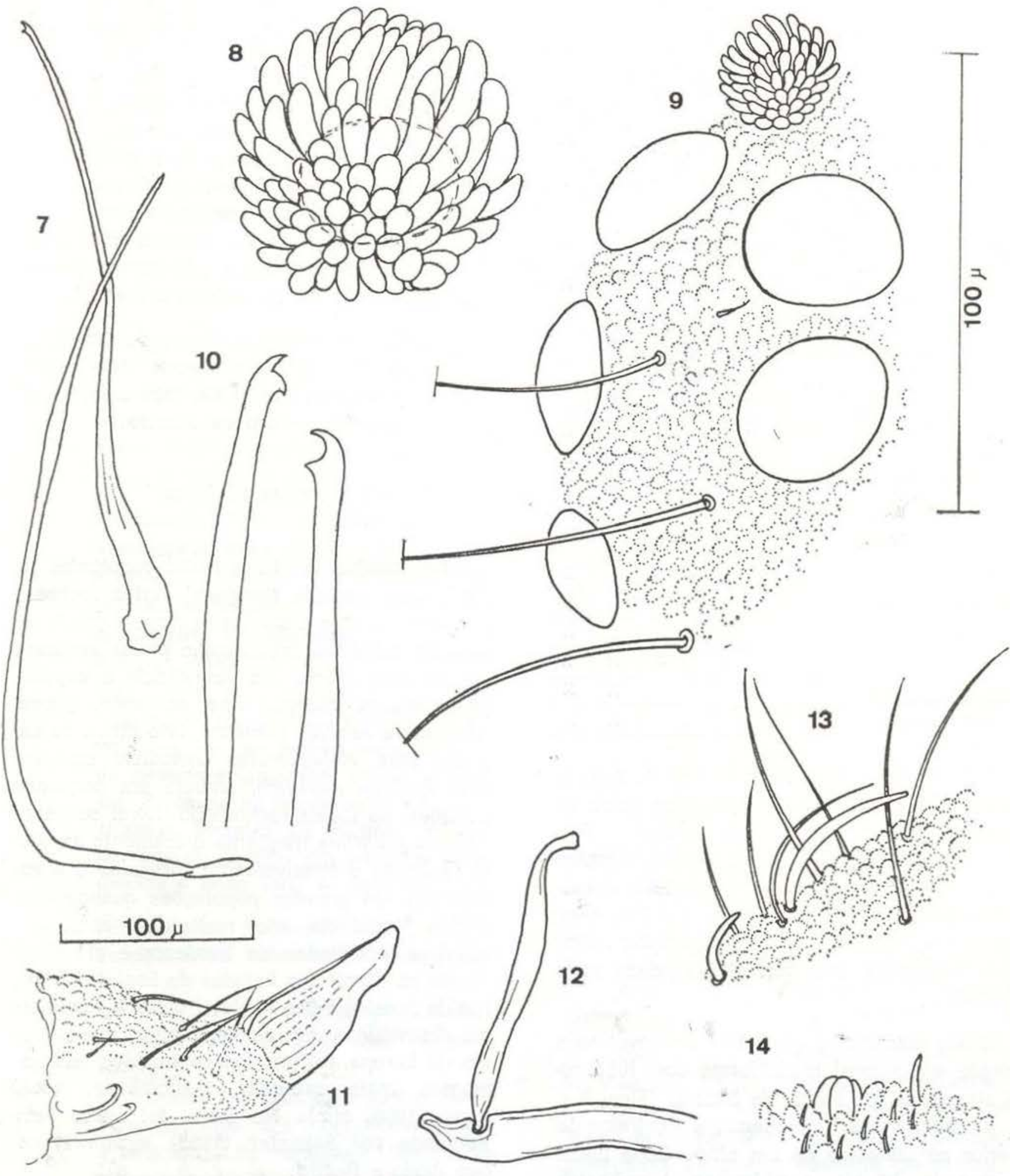

Figs. 7-14 - Neotropiella malkini sp.n.: 7) - Mandibula e maxila; 8) — Orgăo pós-antenal; 9) - Grupo ocular e órgão pós-antenal; 10) - Ápex das mandibulas; 11) - Dens e mucro; 12) - Articulação cardo-fulcro; 13) - Tubos sensitivos da face superior de Ant. IV; 14) - Papila apical de Ant. IV. 


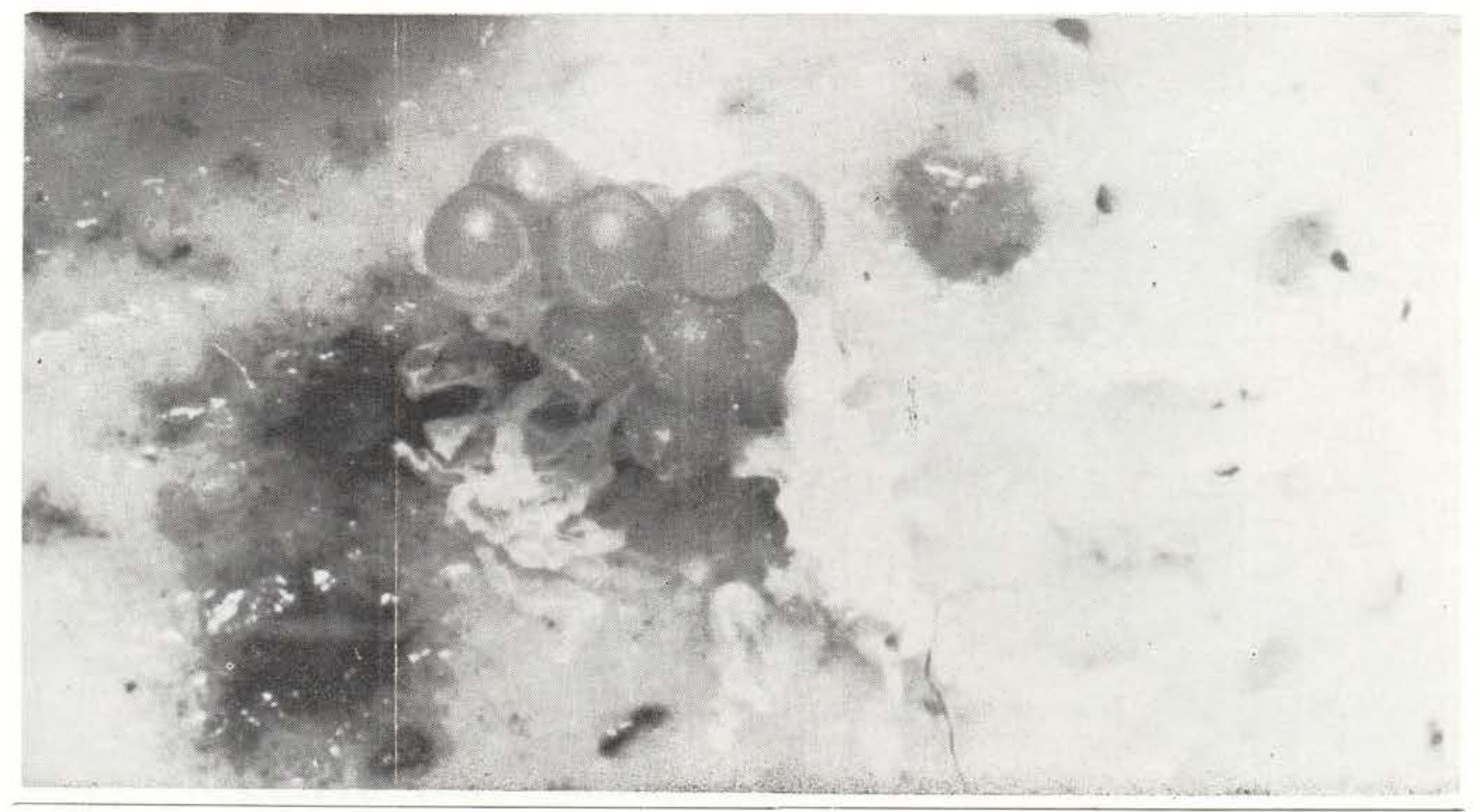

Foto 1 - Halachorutes schusteri: Grupo de ovos postos em cima de enxuvias (em início de desenvolvimento).

tigos destruídos pelo avanço de bancos de areia. Nessas condições abriga-se nos restos de mangue perfurados por turus (Teredo $\mathrm{sp}$.) e outros animars, como acontece em Marudá (Foto 3). Estas populações acompanhando a dinâmica do litoral, são instáveis. Velhos troncos de mangue são arrancados, instalam-se campos de Spartina brasiliensis fixando areias lodosas, novo manguezal se forma onde tinha sido destruído etc., e as colônias de Halachorutes escasseam ou desaparecem de um lugar para se instalarem em novo biótopo favorável.

Os Halachorutes habitam a zona intertidal média totalmente recoberta na preamar e nestas condições necessitam de abrigo, obtido nas anfractuosidades diversas, como troncos de mangue perfurados (Foto 3 ), conchas, cracas, pedaços e blocos de pedra canga que pela sua superficie rugosa e desigual oferecem ótimas condições. Temos observado pequenas cavidades na face inferior dessas pedras que, na preamar, retem ar e funcionam como sino de mergulhador. Estas cavidades estavam completamente forradas de exuvias brancas após os animais as deıxarem, na baixa mar. Conclui-se que as ecdises devem ocorrer si- multaneamente em grupos de animais durante o período de inatividade na maré cheia.

Logo no início da baixa mar os Halachorutes entram em atividade que parece ser mais intensa com dia de sol. Alimentam-se de pequenos animais mortos.

Foi observada a postura em laboratório nos meses de janeiro, fevereiro e março, mas é provável que aconteça durante o ano todo. Animais capturados em Marudá (Pará) foram instalados em cristalizadores de fundo de gesso, com um pouco de areia e lama local, sendo alimentados com moluscos esmagados. Os Halachorutes têm reação de contacto e se apressam uns contra os outros (Foto 2) .

Um pequeno grupo foi recolhido na praia dos pescadores em Marudá no dia 4/III. Nos dias 5 e 6 já apareceram ovos amarelos postos em grupos, medindo $190-200 \mu \mathrm{m}$ de diâmetro. No dia $8 /$ III havia muitos ovos, sendo que uma parte já em desenvolvimento. Muitos ovos eram postos em pequenos grupos sobre as próprias exuvias. Os ovos recém-postos são completamente esféricos e de cor amarela clara; com 2 dias aproximadamente, à temperatu- 

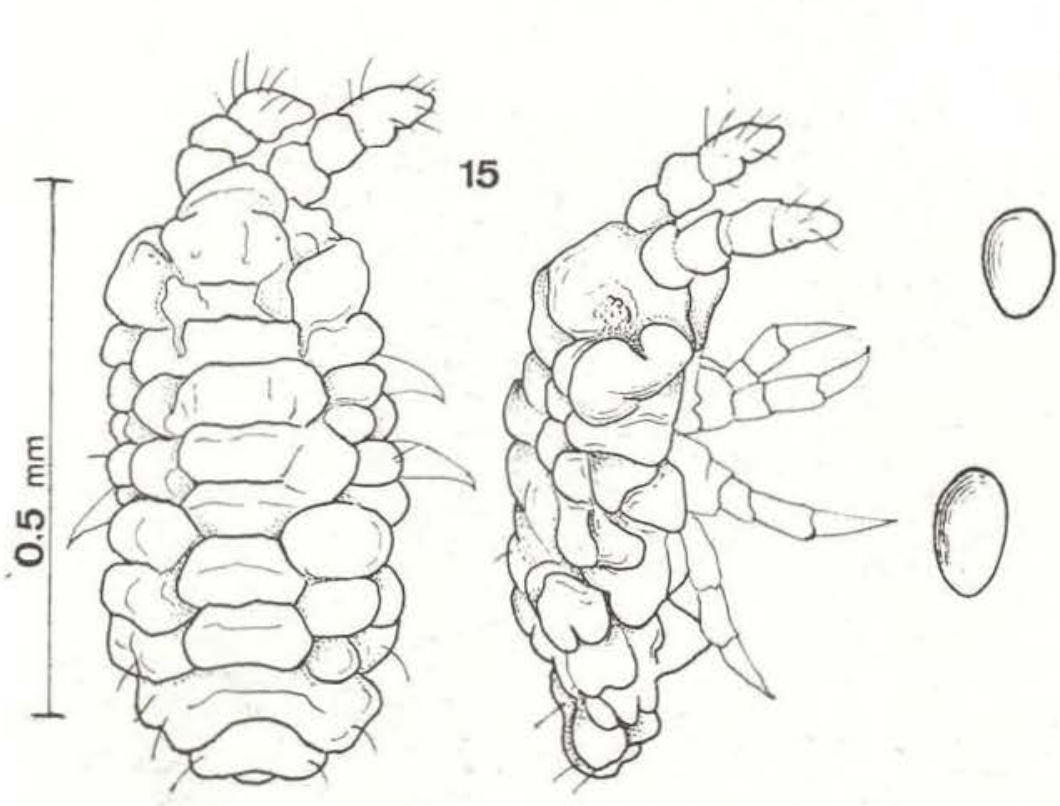

0
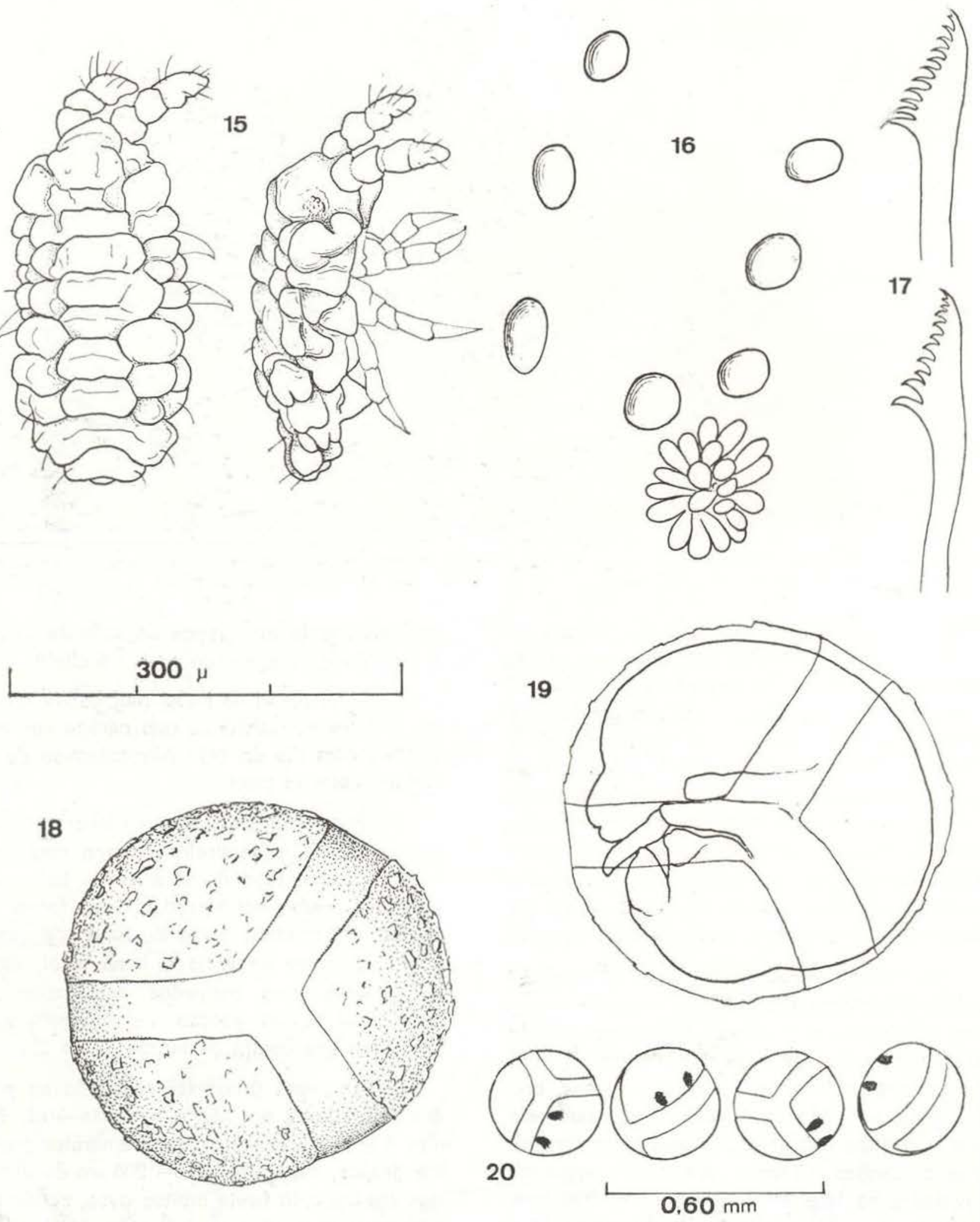

Figs. 15-17 - Neotropiella denisi (Arlé): 15) - Habitus. Exemplares do Alto Xingu; 16) - Grupo ocular e pós-antenal (ex. Xingu); 17) - Mandibulas (ex. Xingu). Figs. 18-20 - Halachorutes schusteri Arl : 18) - Ovo em princípio de desenvolvimento; 19) - Idem, mostrando o embrião; 20) - Ovos pouco antes da eclosão mostrando as manchas oculares do embriāo. 


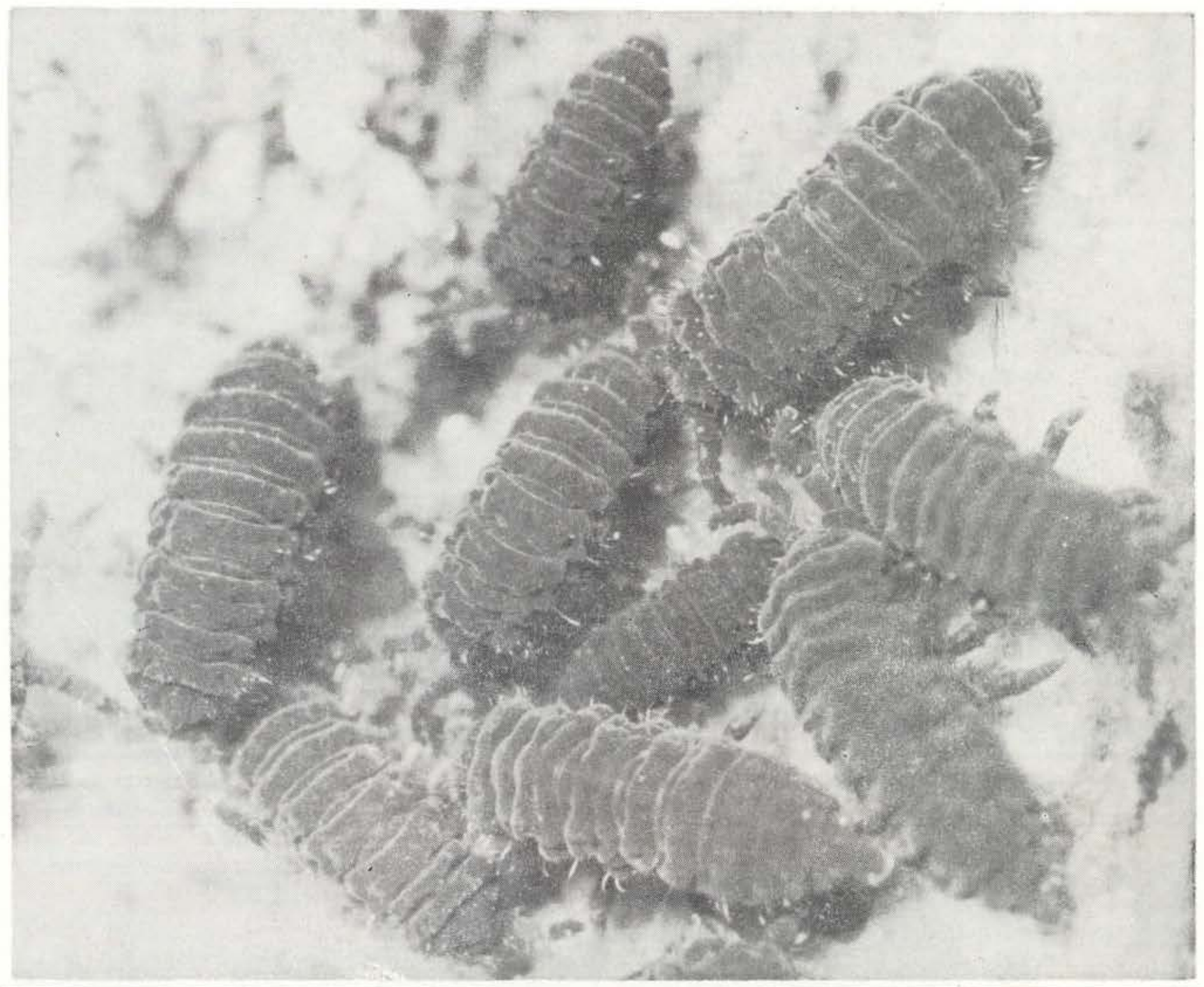

Foto 2 - Halachorutes schusteri: Habitus do animal vivo (Marudá).

ra do laboratório (24-25\%), os ovos aumentam de volume e o córion se divide em 3 gomos iguais (Figs. 18 e 19), medindo neste estado 300 um de diâmetro. Esta divisão em 3 partes obedece a uma polarioade pois a posição do embriâc é constante em relação a esta divisão (Fig. 19). Nos dias 10 a $12 / \mathrm{III}$ apareceram as 2 manchas oculares pretas sempre na mesma posiçāo em relação à divisāo do córion (Fig. 20). No dia 15 eclodiram quase todos os animais.

Nas condições das nossas observações a duração da incubação foi em média de 10 dias. Coservações repetidas no ano seguinte deram resultados semelhantes.

Numa pesquisa realizada nos arredores do Recife foi nos possivel encontrar de novo a
Pseucianurida sawayana. Localizamos colônias fiorescentes no canal que liga a Lagoa do Olho d'água ao mar (Foto 4), nas proximidades da Barra das Jangadas, em 1969. O gênero tem distribuição oriental com espécies na Austrália e a sua presença no Brasil é de grande interesse biogeográfico.

\section{NOTAS E AGRADECIMENTOS}

Limitando-nos às espécies brasileiras, nâo mencionamos os gêneros da tribu Anuridini : Delamarellina Rapoport et Rubio, 1963, Cassagnaurida Salmon 1964 e Notachorudina Cassagnau et Rapoport, 1962, que ocorrem na parte merıdional da América do Sul. Estes gêneros de maxila complexa, são contudo muito próxi- 


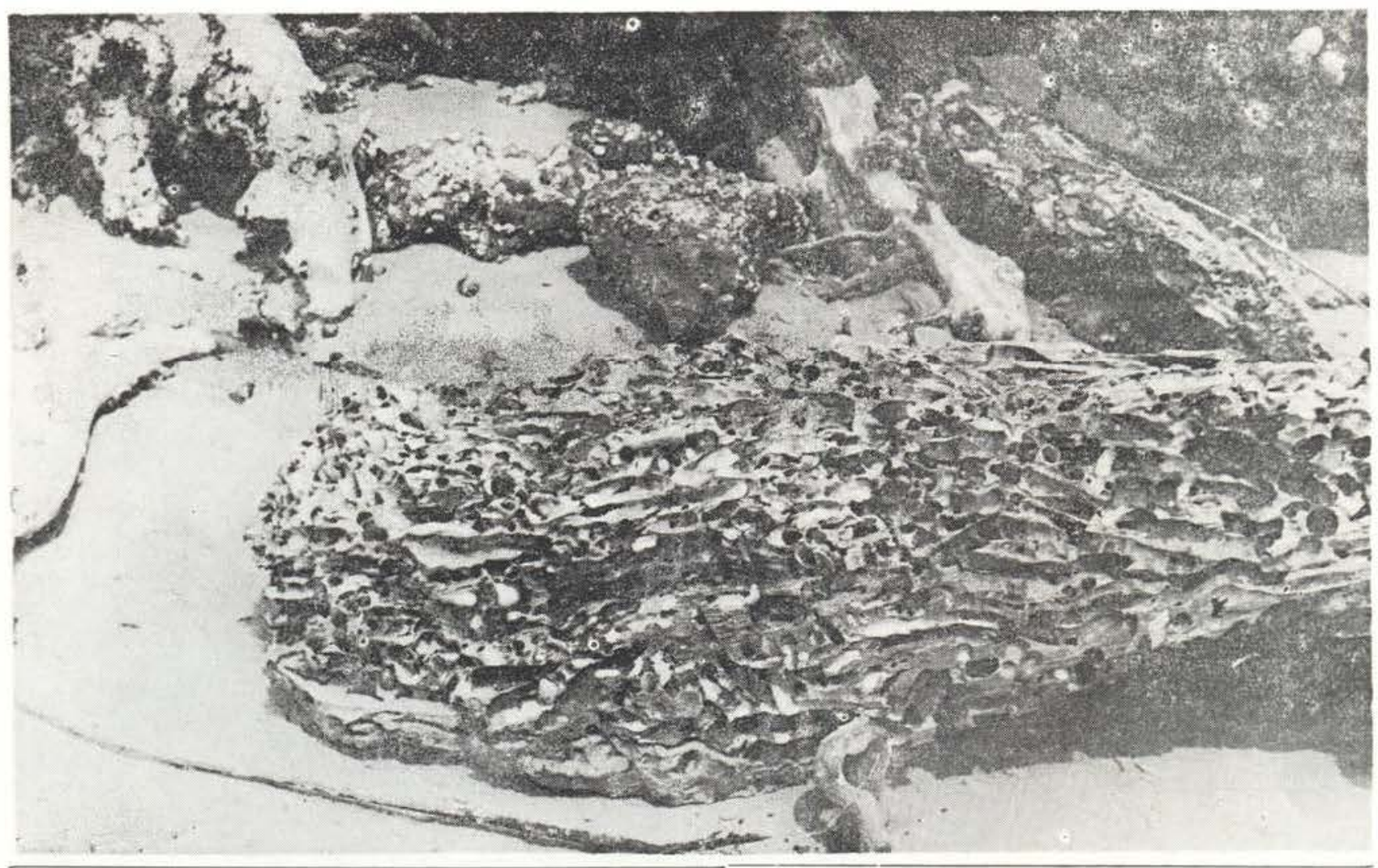

Foto 3 - Halachorutes schusteri: Velho tronco de mangue perfurado por Teredo e servindo de abrigo para os Halachorutes (Praia dos pescadores - Marudá, Pará).

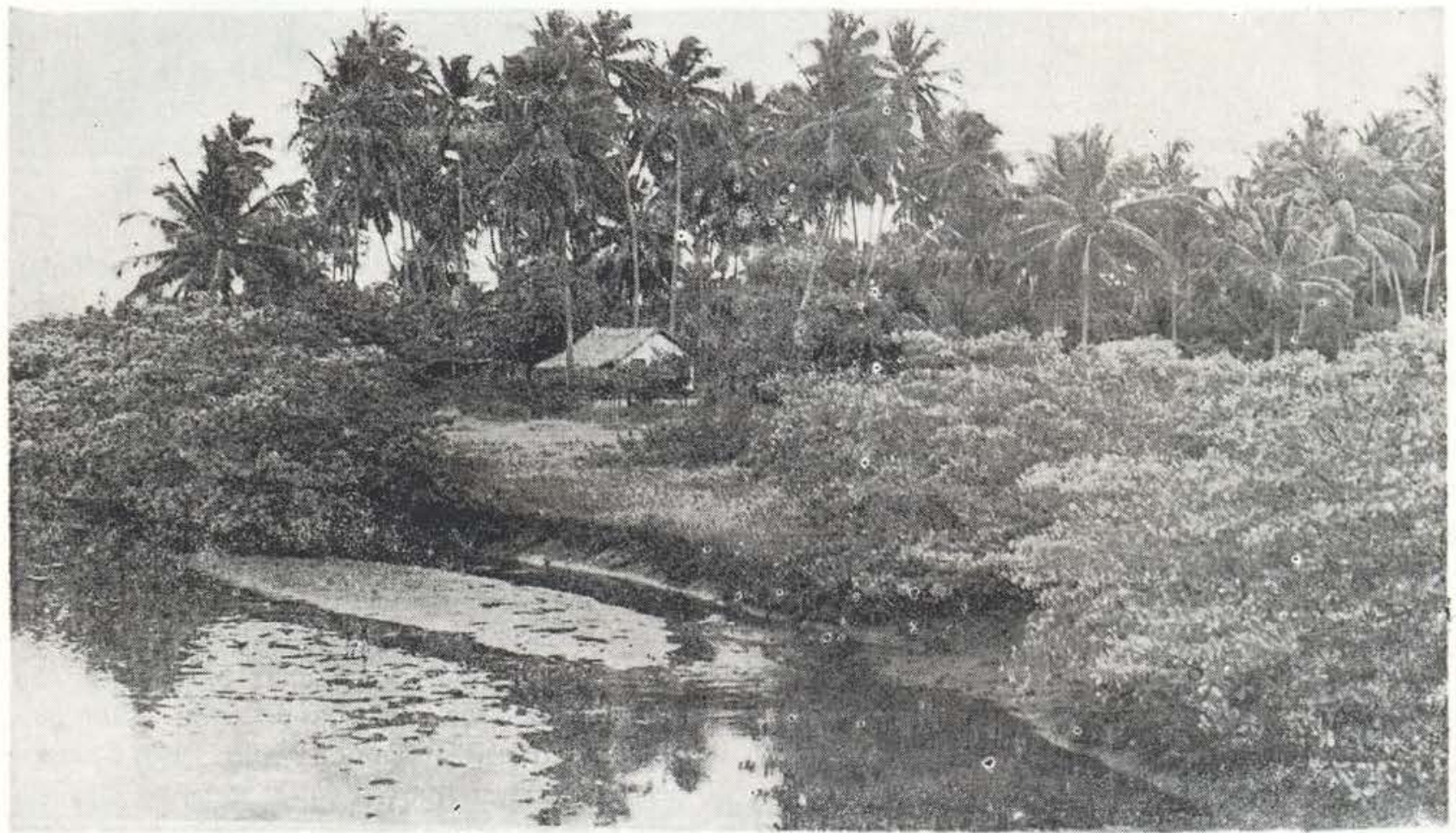

Foto 4 - Biótopo de Pseudanurida sawayana no canal da Lagoa do Olho d'água, Recife (Pernambuco) 1969. As Pseudanurida correm sobre a areia lodosa e raizes de mangue (Laguncularia). 
mos dos gêneros brasileiros tratados aqui. Delarmarellina tem 3 espécies sendo uma na Argentina, uma no Chile e uma na Nova Zelândia.

Agradecemos aqui ao nosso amigo Borys Malkin etno-zoólogo, atualmente residente na Polônia, com quem coletamos no Pará e no Maranhão e de quem recebemos inúmeros Collembola, inclusive lima preciosa coleção da Colômbia.

\section{SUMMARY}

Twenty-two species of Brazilian Pseudachorutinae are recorded. A scheme of geographical distribution for the group in Brazil is given, as far as is presently known. Two species occur in practically all of Brazil; 14 species occur in Amazonia; 12 species in the Southeastern region and six species in the Northeastern region. A new specie of Neotropiella Handschin, 1942 is described from the central Andean region of Colombia (Paramo Purace, $3000 \mathrm{~m}$ height). Observations are given on the bionomics of the thalassic species Halachorutes schusteri Arlé. 1966 from the litoral of Pará State, as well as its development and reproduction in the laboratory. The species Pseudanurida sawayana Schuster, 1965 was reencountered in a mangrove swamp at Recife. Another marine species, Anurida maritima (Guérin), 1838 occurs from Pernambuco State to the southern litoral.

\section{REFERENCIAS BIBLIOGRÁFICAS}

ARLÉ, R.

$$
\begin{aligned}
1939 \text { - } & \text { Novas espécies de Pseudachorutini (Collem- } \\
& \text { bola) do Rio de Janeiro e arredores, Bol. } \\
& \text { Biol. (N.S.) } 4 \text { (1): } 67-72 \\
1943 \text { - } & \text { Deux nouveaux Collemboles du Brésil, Rev. } \\
& \text { Bras. Biol., } 3 \text { (1): 109-112. } \\
1959 \text { - } & \text { Collembola Arthropleona do Brasil Oriental } \\
& \text { e Central. Arq. Mus. Nac. Rio, 49: 155-211. }
\end{aligned}
$$

1966 - Un nouveau Collembole marin du littoral brésilien. Rev. Bras. Biol., 26 (4): 367-372.

1966 - Collemboles d'Amazonie I. Poduromorphes nouveaux ou peu connus et notes biologiques sur Neotropiella carli (Denis) Bol. Mus. Pa. Emílio Goeldi, Belém, n.s. Zool. 60: 1-19.

ARLĖ, R. \& RUFINO, E.

1976 - Contribuição ao conhecimento dos Pseudachorutinae da Amazônia (Collembola). Acta Amazonica, 6 (1): 99-107.

DELAMARE DEBOUTTEVILLE, C.

1951 - Microfaune du sol des pays tempéres et tropicaux. Paris. Hermann ed.

HANDSCHIN, E.

1942 - Materialien zu Revision der Collembolen. Die Gattung Ceratrimeria C.B. sensu Womersley. Verh. Naturforsch. Gesellschaft Basel B. 3: 265-284.

LAWRENCE, P.N.

1971 - A review of the genus Neotropiella Handschin, 1942 (Insecta Collembola) Rex. Ecol. Biol. Sol, 8 (3): 465-470.

MASSOUD, $\mathrm{Z}$.

1967 - Monographie des Neanuridae, Collemboles Poduromorphes a pièces buccales modifiées in: Biol. Amer. Australe, (3) C.N.R.S.,: 1 399 .

SCHUSTER, R.

1965 - Über Ökologie und Artengliederung der thalassobionten Collembolen Fauna Brasilien. Beitrage zur Neotropischen Fauna B., 4 (3): 191-208.

VANZOLINI, P.E.

1973 - Paleoclimates, Relief and Species Multiplication in Equatorial Forests. In: Tropical Forest Ecosystem in Africa and South Ame. rica: A comparative Review. Meggers, Betty J.; Ayensu, Edw. S. \& Donald, W. Duckworth edit. Washington. Smithsonian Inst. Press.

(Aceito para publicação em 12/01/81) 